\title{
Incorporating the maxillary transverse dimension in the treatment plan
}

\author{
P. Bouletreau' ${ }^{1}$, M. Raberin ${ }^{2}$ \\ 1 University Professor, Maxillofacial surgeon \\ 2 University Lecturer, Specialist certified in dentofacial orthopedics
}

\begin{abstract}
Transverse dimension of the maxilla is a key point to consider when elaborating a surgical-orthodontic approach. Though the esthetic impact of transverse maxillary disharmonies is mild, their functional impact is major, with a tendency to underestimate it. The diagnosis of a transverse maxillary disharmony should be made during childhood, pointing out the alveolar part (endo or exoalveoly) and the skeletal part (endo or exognathy) of the dysmorphosis. The authors, an orthodontist and a maxillofacial surgeon, bring their respective look upon transverse maxillary disharmonies and present the various therapeutic means that can be utilized, most of the time through a surgical-orthodontic collaboration. Long-term occlusal stability definitely requires the integration of the maxillary transverse dimension when elaborating a surgical-orthodontic therapeutic approach.
\end{abstract}

KEY WORDS

Dento-maxillary dysmorphosis, endoalveoly, exoalveoly, maxillary endognathy, maxillary exognathy

\section{INTRODUCTION}

The treatment of dentomaxillary dysmorphism requires that therapists employ three-dimensional analyses of dentoskeletal anomalies. This precise spatial understanding is key for achieving the closest thing to an ideal result by combining the class I occlusion with the best possible esthetic result. Sagittal and frontal dimensions are relatively simple to perceive because a trained eye will notice esthetic scars associated with the lack of or excess skeletal growth, manifested in the form of a short or long face or an edentulous or gingival smile, which correspond to excessively concave or convex vertical and profile anomalies in the sagittal direction (fig. 1a and 1b). From a functional perspective, the

Address for correspondence:

Pierre Bouletreau

Service de chirurgie maxillo-faciale

Centre hospitalier Lyon-Sud - 165, Chemin du

Grand Revoyet - 69495 Pierre-Bénite Cedex

E-mail: pierre.bouletreau@chu-lyon.fr

Article received: $10-01-2016$ Accepted for publication: 22-02-2016

This is an Open Access article distributed under the terms of the Creative Commons Attribution License (http://creativecommons.org/licenses/by/4.0), which permits unrestricted use, distribution, and reproduction in any medium, provided the original work is properly cited. 

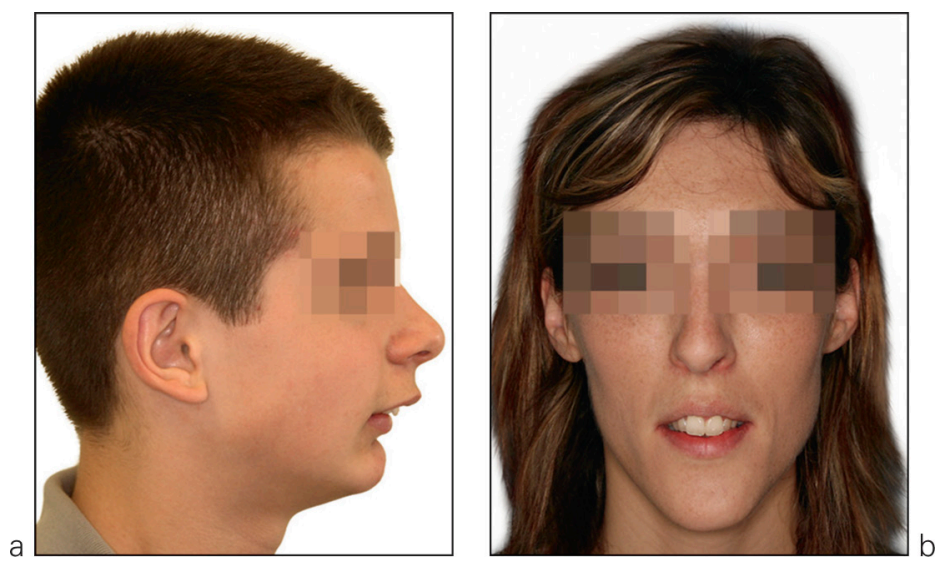

Figure 1

a) A patient with esthetic scars of the class I/ skeletal type caused by mandibular retrognathism. Convex profile. b) A patient with esthetic scars of vertical anterior excess caused by transverse maxillary excess.

Elongated face and excessive uncovering of the maxillary incisors in the resting position.

transverse dimension is crucial; however, it is more difficult to analyze because esthetic consequences are much more discrete, for example, the appearance of excessively visible black triangles in the buccal corridors when smiling in patients with maxillary retrognathism (fig. 2).

Nevertheless, the importance of ensuring a stable correction of a dentoskeletal anomaly in the transverse dimension is currently very important ${ }^{11,12}$. Therefore, it is essential that this dimension is properly integrated into the initial treatment plan.

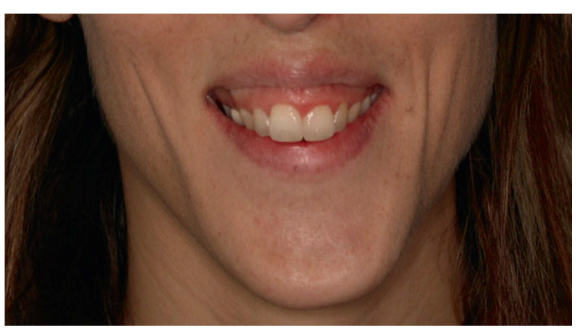

Figure 2

Esthetic scars from asymmetric maxillary prognathism. Excessive black triangle in the right buccal corridor.

\section{THE ORTHODONTIST'S POINT OF VIEW}

The detection of transverse anomalies must be done during childhood, and therapeutic care must be provided from as early as 4 years of age. The consequences to the sagittal and vertical dimensions when growing are catastrophic, but a deficit or excess of the transverse dimension of the max- illary base affects condylar cartilage growth. A unilateral condylar system imposes constraints on the sutural system. Early therapeutic intervention is recommended, and it facilitates the stimulation of the maxillofacial sutural apparatus and the balanced solicitation of condylar cartilages ${ }^{12}$. 


\section{Essential criteria in the diagno- sis of transverse maxillary dysmorphia}

Transverse alveodental or skeletal anomalies have repercussions on facial symmetry. A clinical examination of the symmetry of facial lines and axis of symmetry reflects these and permits the localization of the condition.

Frontal cephalometric analyses according to Ricketts, Sassouni, Grummons, or even Reynecke can quantify and confirm the site of skeletal conditions (fig. 3).

An occlusal examination reveals static and dynamic effects via the presence of cross sections on the unilateral and bilateral sectors. Dentoalveolar compensations can conceal bone- based transverse displacements. The analysis of lateral twisting forces on dental casts can detect spontaneous concealment on the posterior sectors. Alveolar and basal anomalies can coexist. Initial treatment aims to eliminate transverse compensations by reducing the compensatory vestibuloversions of the upper lateral sectors and compensatory linguoversions of the lower molar sectors. Intermolar shifts can lead to patients requiring orthopedic surgery for treating maxillary deficits or excesses.

Conventional or digital casts simulate the correction of the maxillary and mandibular lateral sectors and indicate any residual transverse maxillary deficiency or

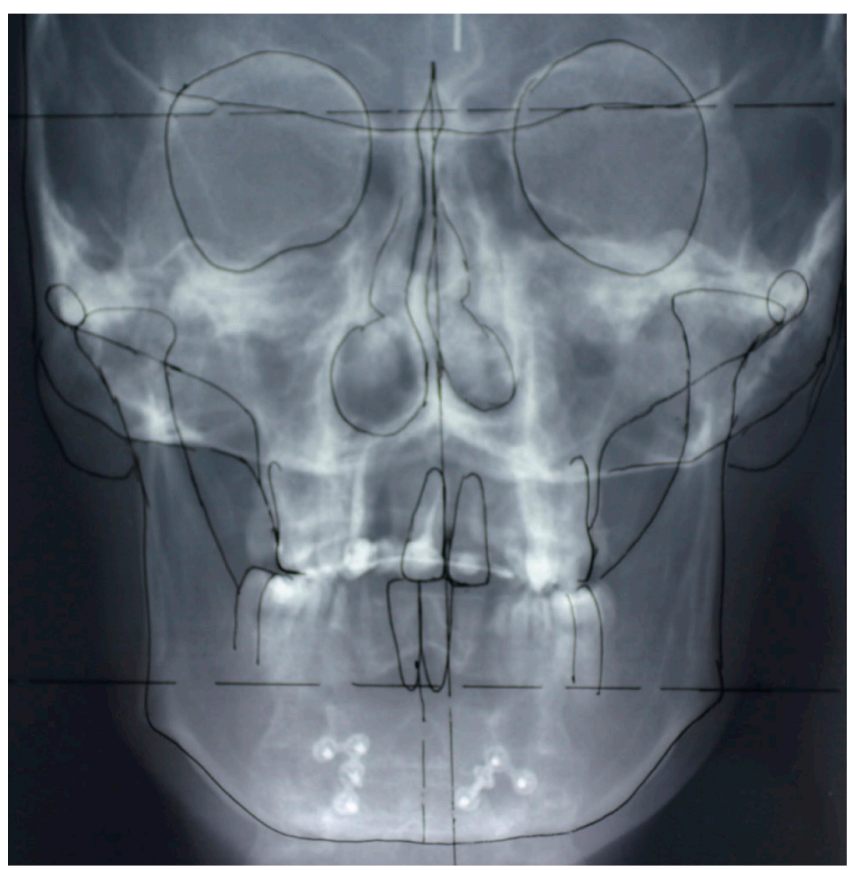

Figure 3

Sassouni facial analysis allowing a rapid analysis of facial symmetry. 
excess. Pairing the examination of the axes of facial symmetry with the location of the median incisor lines using clinical and three-dimensional (3D) radiographic data establishes therapeutic guidelines for orthodontic treatment.

When a deficit exists, these examinations allow a practitioner to make a differential diagnosis between a transverse alveolar deficiency and a transverse maxillary deficiency and to control basal mandibular and/ or functional asymmetry. Transverse treatment is chosen depending on the

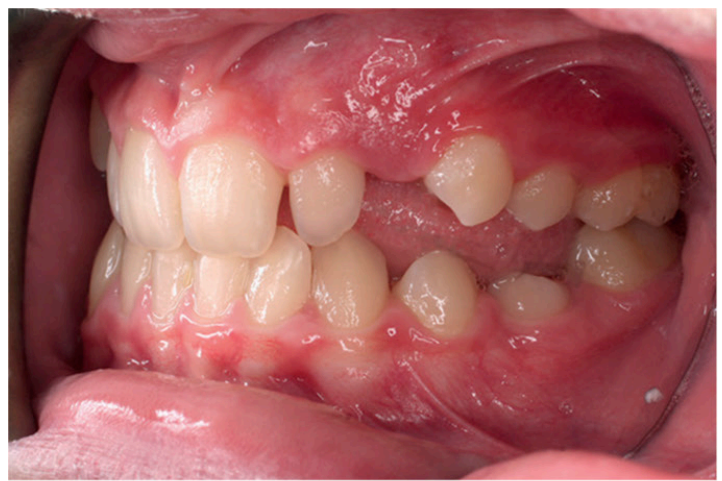

a

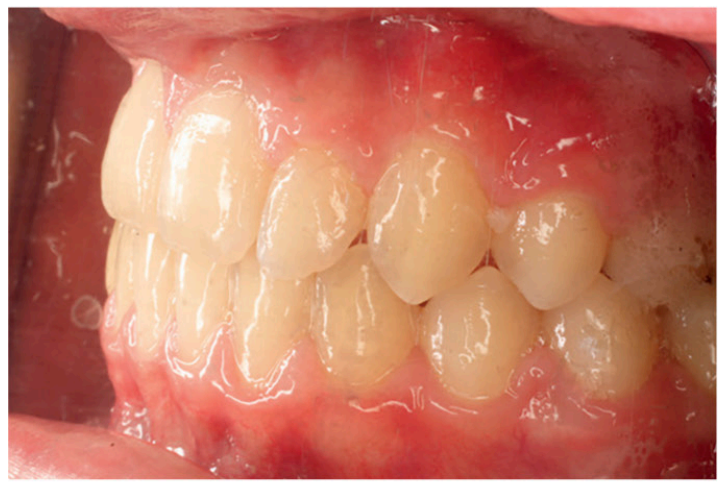

C location and severity and the potential for growth.

The capacity for orthodontic dentoalveolar expansion depends on the periodontal state as well as the histological potential in relation to a patient's age. Surgical expansion by maxillary and/or mandibular distraction associated with multi-attachment treatments contribute to the normalization of the transverse dimension in the presence of a significant transverse deficit, the source of severe overcrowding, or the presence of a fragile periodontium (fig. 4a to $4 d$ ).

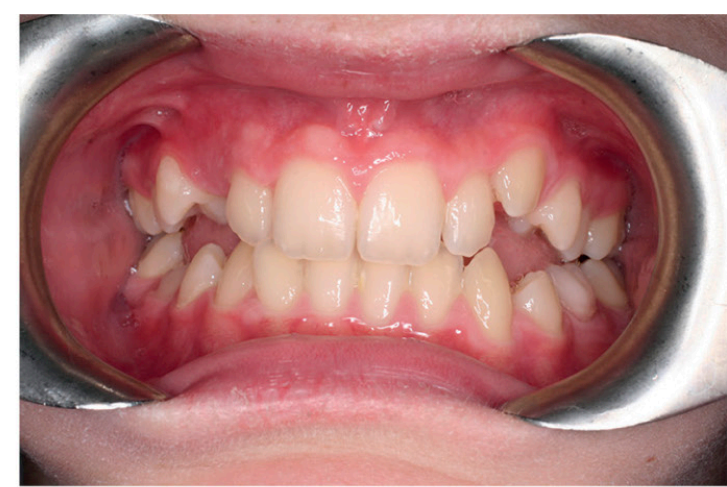

b

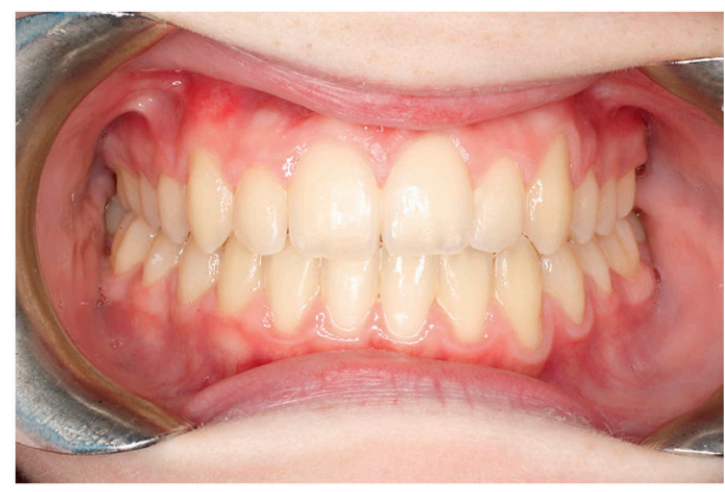

d

Figure 4

A 12-year-old patient showing a moderate transverse alveolar deficit with lateral open bites and upper ectopic canines. Expansion achieved via a multiattachment device without an accessory. 


\section{Correcting transverse maxillary anomalies}

\section{Correcting a transverse alveolar deficiency}

Orthodontic forces of expansion or contraction are applied to the upper arch using a Quad Helix appliance (described by Ricketts), which can expand the lateral sectors. Transverse alveolar deficiency can be seen in milk teeth by attaching it to the second molars and in mixed dentition from the first molars 6 .

The arch is made of Elgiloy steel .032 using a shape memory wire, similar to a "palatal expander" (fig. 5). It must be detachable to allow the molars to move and to control expansion in proportion to the amount of correction achieved.

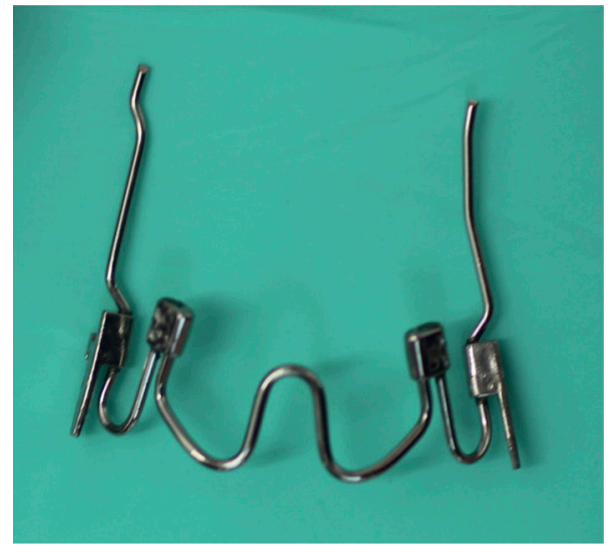

Figure 5

Palatal expander composed of an active part made of NiTi .032 (shape memory alloy) for delivering expansion forces after its compression and Elgiloy steel lateral support bars for shaping the premolar and canine crowns.
An identical system can be put in place on the mandible that permits mandibular expansion depending on the maxillary expansion achieved. A fixed apparatus is favored instead of removable ones because it is not subject to the compliance of patients to the surgical plan 5 .

Vestibular or lingual multi-attachments can treat a transverse maxillary deficit of up to $4 \mathrm{~mm}$ by eliminating compensations that are detrimental to the establishment of centered points and to the stability of occlusal results. Lateral inclination is determined by the information a patient provides and/or the amount of force on orthodontic arch areas. Orthodontic preparation is sometimes necessary to normalize the inclinations of the premolar group for eliminating transverse compensations prior to inserting the Quad Helix. The analysis of casts or digital impressions distributes the transverse actions of fixed orthodontics and of therapeutic complements of alveolar-dental expansion (with an attachment or by intermaxillary disjunction surgery).

\section{Correcting a transverse maxillary deficiency}

In the growth phase and beyond $5 \mathrm{~mm}$, maxillary expansion must be basal and palatovestibular transverse forces must be exerted close to the medial palatal suture by a disjunctor, thus molding the shape and welding the bands to the first molar with extensions plated on the crowns of the first premolars (fig. 6a to 6e). When faced with a major basal deficit, the 

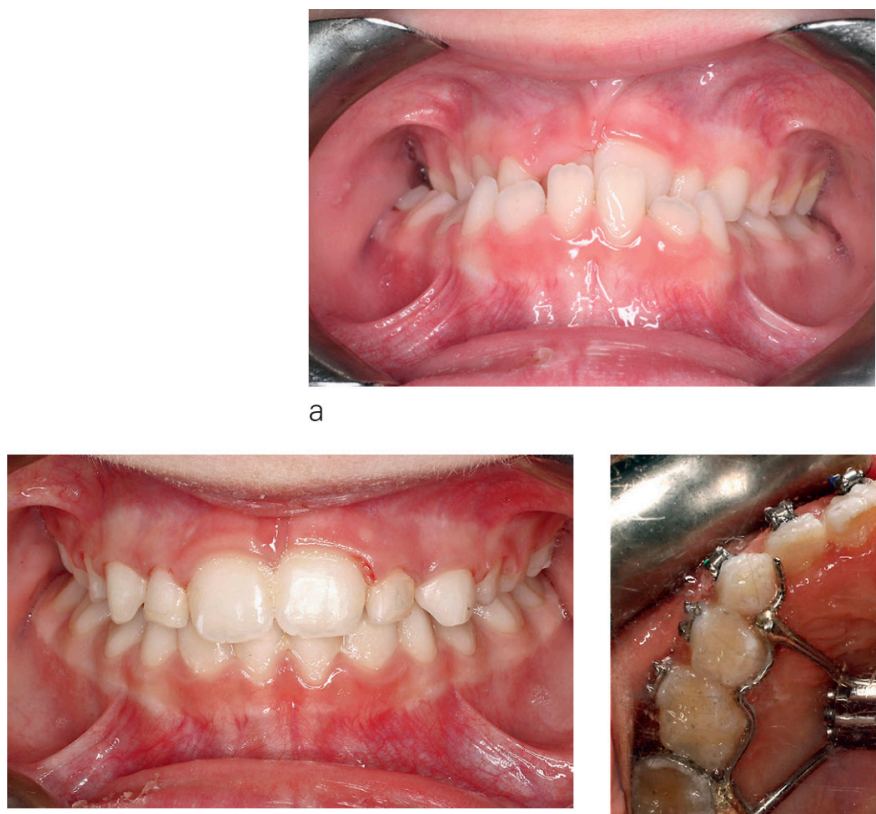

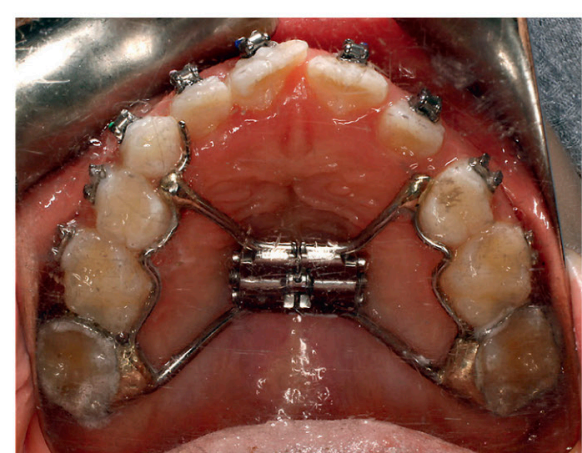

d

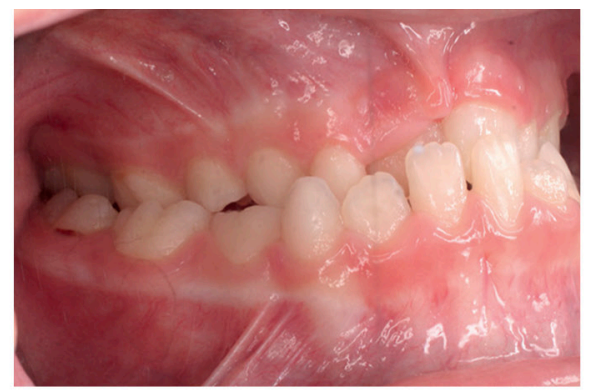

b

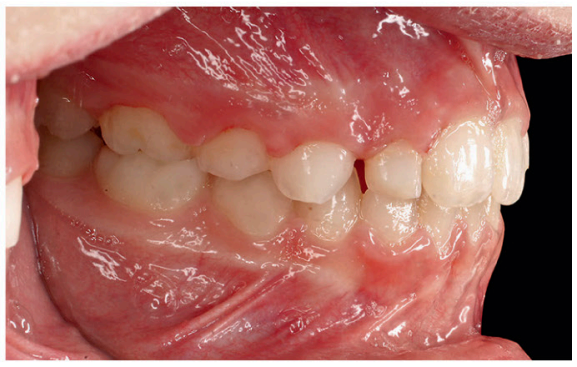

e

Figure 6

Early treatment of a transverse maxillary deficiency. Orthopedic disjunction in a 10-year-old patient treated with a Hyrax disjunctor.

actuator must be close to the intermaxillary suture to reduce the parasitic effects of molar buccoversion. Incidentally, the consensus of the literature is unanimous regarding its functional effects, particularly concerning the re-establishment of nasal respiration and equilibrium in the Eustachian tube ${ }^{12}$. Similar to the treatment of mandibular retrognathism, transverse normalization can be used to prevent sleep apnea.

The Hyrax disjunctor, which is welded onto the first molar bands and extended to the upper canines, requires 1/4-mm activation twice daily. The number of activation turns is calculated based on the deficit measured on casts after simulating lower molar recovery. Depending on the type of actuator used and the amount of expansion to be gained, a second actuator is an option; this can be used either after the first phase of expansion or during treatment after the bone mineralization phase of the distended cartilaginous suture or even in the event of a secondary deficit of transverse maxillary growth, which may have appeared during treatment.

Orthopedic distraction is possible up to mediopalatal and pterygopalatal sutures either 16 years of age for boys or 14 years of age for girls ${ }^{1}$. Afterwards, a 3D cone-beam radiological examination of the median suture is recommended to avoid exerting heavy forces on a suture that can be either totally or partially fused by synostosis. This will induce 


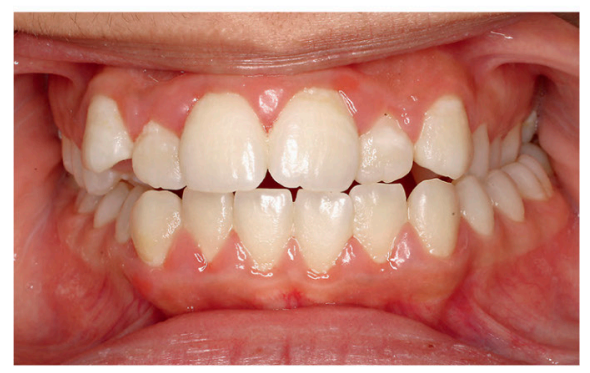

a

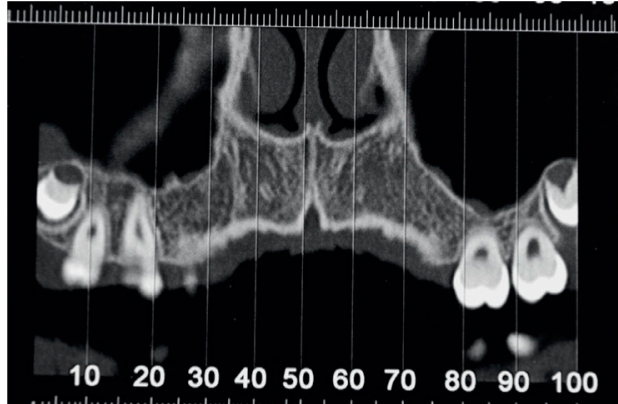

b

Figure 7

A 14-year-old patient showing a transverse alveolar deficiency associated with left laterodeviation. A 3D examination in search of absent synostosis zones of the median palatal suture for possible basal expansion.

fenestrations by breaking lateral alveolar cortical bones (fig. 7). These iatrogenic effects can also be observed during orthodontic expansion via high-strength bows, where the shape and transverse dimensions of the initial arch are ignored. Assisted surgical expansion of this suture is recommended in the presence of advanced synostosis ${ }^{9,11}$.

The contention phase of transverse expansion must be maintained beyond $3 \mathrm{~mm}$ for a minimum of 6 months, either by a disjunctor or the Quad Helix, which is usually maintained even after the active phase, with a fixed contention device attached to the transpalatal arch. A hypercorrection is often necessary because of parasitic versions that are eliminated after inserting the expansion appliance? ${ }^{7}$

\section{THE SURGEON'S POINT OF VIEW}

From a surgical perspective, there are many therapeutic approaches available for correcting a skeletal anomaly

\section{Functional Education}

A transverse alveolar deficiency causes functional mandibular laterodeviation, which corresponds to Cauhépé-Fieux syndrome. The etiology of this syndrome can be unilateral mastication or bad habits such as deficient and lecherous sucking.

The rehabilitation of alternate bilateral mastication and equilibrium among the masticator muscles during swallowing affect the treatment timeline during the contention phase after maxillary expansion that occurs once the expansion devices have been removed. During the expansion phase or contention phase, osteopathic treatment allows equal distribution of transverse forces on the sutural system and an improvement in the histological response.

of the transverse dimension ${ }^{3}$. The surgical strategy for choosing the best therapeutic route can be adopted at 
the beginning of treatment based on of the following three distinct dysmorphic frameworks.

\section{Severe maxillary prognathism greater than $7 \mathrm{~mm}$}

In this situation, we must first conceive an initial maxillary disjunction. This will be handled by orthopedic specialists before patients reach 12 years of age and by orthopedic-surgical specialists after they reach 14 years of age. Between the ages of 12 and 14 years, it is acceptable to begin correction for maxillary prognathism using only orthopedic techniques but practitioners should be extremely cautious of how the treatment proceeds. At instances where the process of disjunction is stagnated or at the slightest sign of iatrogenic complications such as cortical radicular fenestration, orthopedic treatments should be halted and orthopedic-surgical treatment should be considered. The ages indicated above must be adjusted depending on individual residual growth and the degree of fusion of the intermaxillary suture in each patient.

The focus of performing maxillary disjunction with an orthodontic-surgical treatment plan is two-fold. On the one hand, it facilitates orthodontic preparation without tooth extractions in the event of anterior overcrowding of teeth. On the other hand, it corrects the skeletal transversal dimension, albeit imperfectly, at the start of orthodontic preparation and allows us to approach the second surgical stage with a more favorable technique. It is well known that the skeletal correction of an upper maxillary prognathism greater than $7 \mathrm{~mm}$ via palatal expansion during Lefort 1 osteotomy is associated with an extremely high rate of relapse, which has been deemed "problematic" by Proffit ${ }^{10}$. Therefore, we can conclude that a treatment plan that would propose one stage of surgical correction of the transverse dimension at the end of orthodontic preparation is inadequate if the maxillary prognathism is initially measured to be greater than $7 \mathrm{~mm}$.

\section{Moderate maxillary prognathism is approximately $4-7 \mathrm{~mm}$}

In this situation, correcting the transverse dimension can be conventionally contemplated once orthodontic preparation is complete. Maxillary expansion will be conducted according to the Lefort 1 palatal osteotomy technique. If the scale of correction to the transverse dimension is significant, it would be advisable to multiply palatal osteotomy lines to better distribute palatal mucosal tensions (fig. 8). Piezotome is useful because it is more precise with osteotomy lines. A treatment plan integrating transverse dimension correction has been well detailed ${ }^{10}$, and we must also

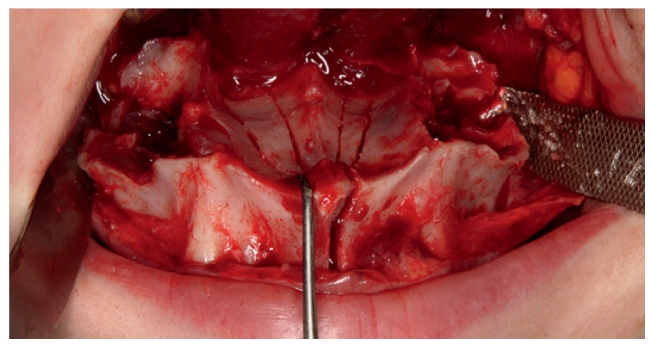

Figure 8

Superior operative view of the maxillary prognathism after multiple palatal osteotomies have been conducted via Piezotome. 
consider that the average prolonged contention phase of the said correction has a strong tendency to relapse. As per the preferences of practitioners, a transpalatal arch or surgical splint can be used and should be maintained for 4 to 6 months postoperatively.

\section{Moderate maxillary prognathism less than $4 \mathrm{~mm}$}

This situation requires correction even if it is less significant because an occlusion balanced in the three planes of space is paramount to the long-term stability of orthodontic treatment ${ }^{8}$. Therefore, it is necessary to analyze the esthetic equilibrium of the face when choosing the appropriate option for correcting the transverse dimension.

If it is also desirable to correct the maxillary spatial position in the frontal plane (anterior vertical excess or deficiency), sagittal plane (mandibular retrognathism), or axial plane (point 1 deviation), correction of the transverse dimension will be easily achieved in the surgical stage of maxillary osteotomy (cf. supra).

\section{CONCLUSION}

The integration of the transverse dimension into the orthodontic-surgical treatment plan has little impact from an esthetic point of view, but it is paramount for maintaining long-term occlusal stability. Careful clinical analysis of the occlusion at the start of treatment must lead to a precise diagnosis of the type and size of the transverse anomaly: transverse alveolar deficiency

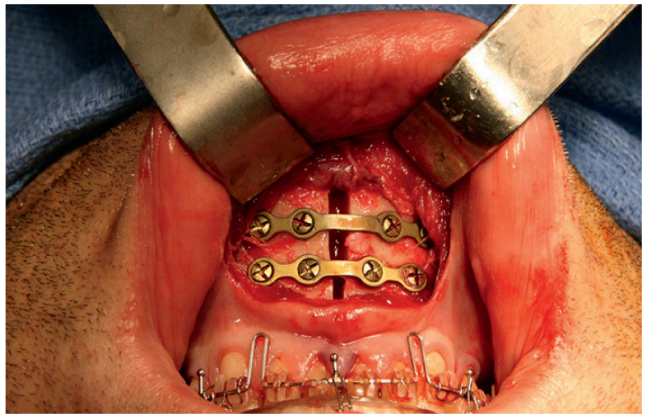

Figure 9

Operative view of mandibular contraction permitting the reduction in posterior mandibular intermolar distance.

On the contrary, if all maxillary actions seem unnecessary outside the correction of the maxillary prognathism, mandibular contraction must be considered ${ }^{2}$. If mandibular propulsion must be performed to correct a class II and a maxillary prognathism less than $4 \mathrm{~mm}$ that still exists even at the end of orthodontic preparation, it is acceptable to propose mandibular contraction concomitant with sagittal osteotomy of mounting mandibular branches. In this way, the surgical procedure is simplified and maxillary action is avoided (fig. 9).

or transverse maxillary deficiency. This analysis will determine the treatment schedule (one or two surgical stages) as well as the technical means that will be employed: arches, anchorage screws, and orthopedic or orthopedicsurgical expansion.

Conflict of interest: The authors declare that they have no conflict of interest. 


\section{BIBLIOGRAPHY}

1. BaccettiT, Franchi L, Cameron CG, McNamara JA Jr. Treatment timing for rapid maxillary expansion. Angle Orthod 2001;71:343-50.

2. Bouletreau P. [Mandibular constriction: managing the skeletal transversal discrepancies]. Rev Stomatol Chir Maxillofac 2009;110:198-201.

3. Bouletreau P, Paulus C. Surgical correction of transverse skeletal abnormalities in the maxilla and mandible. Int Orthod 2012;10:261-273.

4. Ciambotti C, Ngan P, Durkee M, Kohli K, Kim H. A comparison of dental and dentoalveolar changes between rapid palatal expansion and nickel-titanium palatal expansion appliances. Am J Orthod Dentofacial Orthop 2001;119:11-20.

5. Hilgers JJ. A palatal expansion appliance for non-compliance therapy. J Clin Orthod 1991;25:491-497.

6. Mosleh MI, Kaddah MA, Abd ElSayed FA, ElSayed HS. Comparison of transverse changes during maxillary expansion with 4-point bone-borne and tooth-borne

7. maxillary expanders. Am J Orthod Dentofacial Orthop 2015;148:599-607.

8. Moussa R, O'Reilly MT, Close JM. Long-term stability of rapid palatal expander treatment and edgewise mechanotherapy. Am J Orthod Dentofacial Orthop 1995; 108:478-488.

9. Philippe J. [The multiple causes of relapse]. Orthod Fr 2005;76:183-186.

10. Pogrel MA, Kaban LB, Vargervik K, Baumrind S. Surgically assisted rapid maxillary expansion in adults. Int J Adult Orthodon Orthognath Surg 1992;7:37-41.

11. Proffit WR, Turvey TA, Phillips $C$. The hierarchy of stability and predictability in orthognathic surgery with rigid fixation: an update and extension. Head Face Med 2007;3:21.

12. Raberin M. [Orthodontic implications in the correction of the transverse dimension in orthognathic surgery]. Rev Stomatol Chir Maxillofac 2001;102:325-333.

13. Raberin M. [Pathology and treatment of the transverse dimension in the mixed dentition. Impact on muscular equilibrium]. Orthod Fr 2001;72:131-41,199-213. 